\title{
Exploring Energy-Time Entanglement Using Geometric Phase
}

\author{
Anand Kumar Jha, Mehul Malik, and Robert W. Boyd \\ The Institute of Optics, University of Rochester, Rochester, New York 14627, USA
}

(Dated: March 5, 2018)

\begin{abstract}
Using the signal and idler photons produced by parametric downconversion, we report an experimental observation of a violation of the Bell inequality for energy and time based purely on the geometric phases of the signal and idler photons. We thus show that energy-time entanglement between the signal and idler photons can be explored by means of their geometric phases. These results may have important practical implications for quantum information science by providing an additional means by which entanglement can be manipulated.
\end{abstract}

Published in: Phys. Rev. Lett. 101, 180405 (2008) 10.1103/PhysRevLett.101.180405

PACS numbers: 03.65.Ud, 03.65.Vf, 03.67.Bg

Geometric phase, or Berry's phase, is the phase acquired by a system when it is transported around a closed circuit in an abstract space [1]. The manifestation of this phase in polarization optics is also known as Pancharatnam phase, which is the phase acquired by a photon field when its polarization is taken through a closed circuit on the Poincaré sphere [2-4]. Pancharatnam phase has been observed both at high light levels [5-7] and at a single photon level [8]. Effects of Pancharatnam phase in two-photon interference, using the signal and idler photons produced by parametric downconversion (PDC), have also been studied in many different situations [9-13].

Bell's inequality [14] was derived in order to show that any local hidden variable interpretation [15] of quantum mechanics is incompatible with the statistical predictions of quantum mechanics. Clauser, Horne, Shimony and Holt (CHSH) generalized Bell's inequality so that it could be applied to realizable experiments [16]. Since then, using the signal and idler photons produced by PDC, violations of the generalized forms of Bell's inequality have been observed for various degrees of freedom including polarization [17, 18], phase and momentum [19], and energy and time $[20-22]$. In recent years, using hyperentangled states, even simultaneous violations of Bell inequalities for more than one degrees of freedom have been reported [23-26]. In addition to proving the impossibility of local hidden variable interpretations of quantum mechanics, a violation of Bell's inequality, based on a certain degree of freedom, verifies entanglement and guarantees that it can be exploited through that particular degree of freedom.

Bell inequality for energy and time was suggested by J. D. Franson using an experimental scheme commonly known as the Franson interferometer [20]. Franson's scheme for violating a Bell inequality requires changing the phases of the signal and idler photons in one of the interfering alternatives. In all the experimental realizations of Franson's scheme so far, the phases of the signal and idler photons have been changed by adjusting their dynamic phases, i.e., by adjusting their optical path lengths
$[21,22]$. Therefore, all these previous violations can be said to be the dynamic phase-based violations of Bell inequality for energy and time.

In this paper, we show that the Bell inequality for energy and time can also be violated using geometric phases of the signal and idler photons and that therefore the energy-time entanglement can be explored using their geometric phases. Throughout this paper, we use Ref. [27] as our theoretical framework.

Consider the double-pass setup [28] shown in Fig. 1(a). A cw Ar-ion laser operating at $363.8 \mathrm{~nm}$ is used as a pump to produce degenerate type-I parametric downcon-

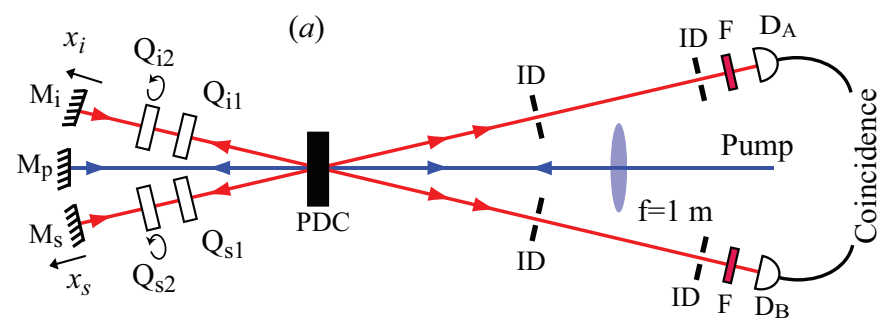

(b)

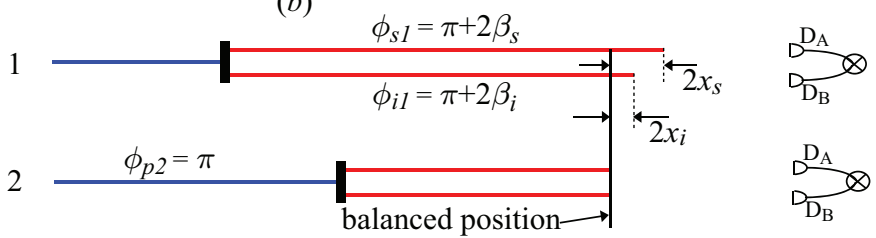

FIG. 1: (color online). (a) Schematic of the experimental setup. $\mathrm{Q}_{\mathrm{s} 1}$ and $\mathrm{Q}_{\mathrm{i} 1}$ are quarter-wave plates with their optic axes oriented at $45^{\circ}$ from the horizontal polarization direction. $\mathrm{Q}_{\mathrm{s} 2}$ and $\mathrm{Q}_{\mathrm{i} 2}$ are rotatable quarter-wave plates with their optic axes oriented at angles $135^{\circ}+\beta_{s}$ and $135^{\circ}+\beta_{i}$ from the horizontal polarization direction respectively. $\mathrm{F}$ is an interference filter with 10-nm bandwidth, centered at $727.6 \mathrm{~nm}$; ID is an iris diaphragm. The signal and idler photons are collected into multimode fibers and detected using two avalanche photodiodes. A weak lens $(\mathrm{f}=1 \mathrm{~m})$ focuses the pump beam onto the pump mirror $\mathrm{M}_{\mathrm{p}}$. (b) Two-photon path diagrams illustrating how the geometric phases $2 \beta_{s}$ and $2 \beta_{i}$ influence the two-photon interference. 
verson (PDC). The pump is vertically polarized and the downconverted photons are both horizontally polarized. The quarter-wave plates $\mathrm{Q}_{\mathrm{s} 1}$ and $\mathrm{Q}_{\mathrm{i} 1}$ are arranged with their optic axes oriented at angle $45^{\circ}$ from the horizontal polarization direction, while the rotatable quarter-wave plates $\mathrm{Q}_{\mathrm{s} 2}$ and $\mathrm{Q}_{\mathrm{i} 2}$ are arranged with their optic axes oriented at angles $135^{\circ}+\beta_{s}$ and $135^{\circ}+\beta_{i}$ from the horizontal polarization direction respectively. In this setup there are two alternative pathways - represented by the twophoton path diagrams in Fig. 1(b) - by which the pump photon gets downconverted and the downconverted photons get detected at single-photon detectors $D_{\mathrm{A}}$ and $D_{\mathrm{B}}$. In alternative 1 , a pump photon gets downconverted in its forward pass and the downconverted signal and idler photons reach the two detectors $D_{\mathrm{A}}$ and $D_{\mathrm{B}}$ after passing through the quarter-wave plates and getting reflected from the signal $\left(\mathrm{M}_{\mathrm{S}}\right)$ and idler $\left(\mathrm{M}_{\mathrm{i}}\right)$ mirrors. In alternative 2, a pump photon gets downconverted after getting reflected from the pump mirror $\left(\mathrm{M}_{\mathrm{p}}\right)$ and the downconverted photons directly reach their respective detectors.

In alternative 1 , a horizontally polarized signal photon passes through the quarter-wave plates $\mathrm{Q}_{\mathrm{s} 1}$ and $\mathrm{Q}_{\mathrm{s} 2}$, gets reflected back from the signal mirror $M_{S}$ and retraces its path through the quarter-wave plates $\mathrm{Q}_{\mathrm{s} 2}$ and $\mathrm{Q}_{\mathrm{s} 1}$. It becomes horizontally polarized after completing the loop but in this process it acquires a geometric phase equal to $2 \beta_{s}$, as illustrated in Fig. 2. This phase is in addition to the dynamic phase that the signal photon acquires. Similarly, the idler photon acquires a geometric phase equal to $2 \beta_{i}$ in alternative 1 . In the balanced position of the setup in Fig. 1(a), the optical path lengths between the crystal and each of the three mirrors are assumed to be equal, to about $15 \mathrm{~cm}$. The displacements of the signal and idler mirrors from the balanced position are denoted by $x_{s}$ and $x_{i}$ respectively.

We recall the definitions in Ref. [27] for the two length parameters $\Delta L$ and $\Delta L^{\prime}$ and one phase parameter $\Delta \phi$, which are given as:

$$
\begin{aligned}
\Delta L & \equiv l_{1}-l_{2}=\left(\frac{l_{s 1}+l_{i 1}}{2}+l_{p 1}\right)-\left(\frac{l_{s 2}+l_{i 2}}{2}+l_{p 2}\right) \\
\Delta L^{\prime} & \equiv l_{1}^{\prime}-l_{2}^{\prime}=\left(l_{s 1}-l_{i 1}\right)-\left(l_{s 2}-l_{i 2}\right) \\
\Delta \phi & \equiv\left(\phi_{s 1}+\phi_{i 1}+\phi_{p 1}\right)-\left(\phi_{s 2}+\phi_{i 2}+\phi_{p 2}\right) .
\end{aligned}
$$

Here the subscripts $p, s$ and $i$ stand for pump, signal and idler respectively; $l$ denotes the optical path-length travelled by a photon; and $\phi$ stands for phases other than the dynamic one such as phase acquired due to reflections, geometric phase etc. Thus, $l_{s 1}$ denotes the path length travelled by the signal photon in alternative 1, etc.

The complete two-photon state $\left|\psi_{\text {tp }}\right\rangle$ produced by the double pass setup of Fig. 1(a) is the coherent sum of the two-photon states produced in alternatives 1 and 2, and using the definitions of Eq. (1) it can be written as

$$
\left|\psi_{\mathrm{tp}}\right\rangle=\left|\psi_{\mathrm{tp}}\right\rangle_{1}+e^{-i\left(k_{0} \Delta L+\Delta \phi\right)}\left|\psi_{\mathrm{tp}}\right\rangle_{2} .
$$

(a)

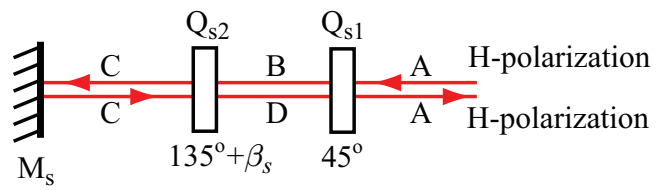

(b)

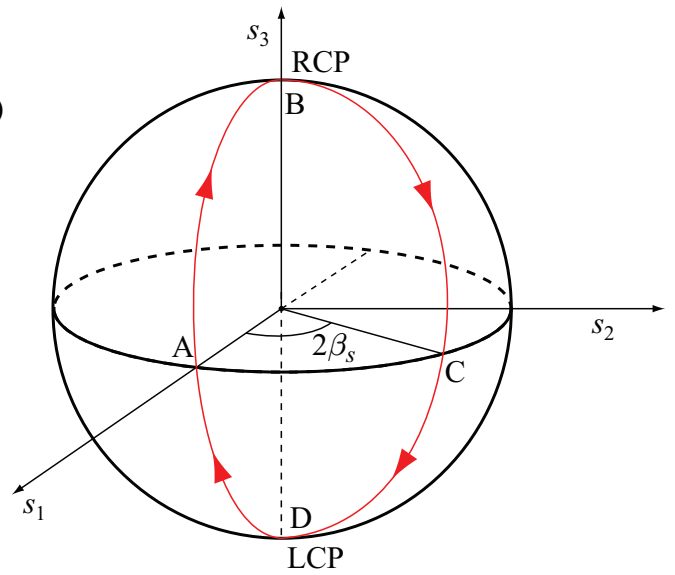

FIG. 2: (color online). (a) The signal photon path ABCDA through the two quarter-wave plates $\mathrm{Q}_{\mathrm{s} 1}$ and $\mathrm{Q}_{\mathrm{s} 2}$, in alternative 1. (b) Poincaré-sphere representation of polarization states of the signal photon corresponding to path ABCDA. A signal photon acquires a phase $2 \beta_{s}$ in going through path ABCDA, which is equal to half of the solid angle subtended by the closed circuit ABCDA on the Poincaré sphere.

Here $\left|\psi_{\mathrm{tp}}\right\rangle_{1(2)}$ is the two-photon state is alternative 1(2); $k_{0}$ is the mean vacuum wavevector magnitude of the pump wave and $k_{0} \Delta L+\Delta \phi$ is the relative phase of the two-photon state in alternative 2 with respect to the twophoton state in alternative 1 . Interference is observed in the coincidence count rate $R_{\mathrm{AB}}$ of detectors $D_{\mathrm{A}}$ and $D_{\mathrm{B}}$, which can be calculated using the general expression derived in Ref.[27]:

$$
R_{\mathrm{AB}}=C\left[1+\gamma(\Delta L) \gamma^{\prime}\left(\Delta L^{\prime}\right) \cos \left(k_{0} \Delta L+\Delta \phi\right)\right] .
$$

Here $\mathrm{C}$ is a constant; $\gamma(\Delta L)$ is the pump correlation function with a width equal to $l_{\mathrm{coh}}^{p}$, the coherence length of the pump, which in our case is about $5 \mathrm{~cm} ; \gamma^{\prime}\left(\Delta L^{\prime}\right)$ is the signal-idler correlation function with a width equal to $l_{\text {coh }}$. The signal-idler coherence length $l_{\text {coh }}$ is determined by the widths of the interference filters and by the sizes of the iris diaphragms, and is equal to about $100 \mu \mathrm{m}$ in our case. From the two-photon path diagram [Fig. 1(b)] and using Eq. (1), we find that $\Delta L=x_{s}+x_{i}$, $\Delta L^{\prime}=2 x_{s}-2 x_{i}$ and $\Delta \phi=2 \beta_{s}+2 \beta_{i}+\pi$. Assuming $|\Delta L| \ll l_{\text {coh }}^{p}$ and $\left|\Delta L^{\prime}\right| \ll l_{\text {coh }}$, Eq. (3) simplifies to

$$
R_{\mathrm{AB}}=C\left\{1-\cos \left[k_{0}\left(x_{s}+x_{i}\right)+2 \beta_{s}+2 \beta_{i}\right]\right\} .
$$

When the geometric phase $2 \beta_{s}+2 \beta_{i}$ is held fixed, the variation of the coincidence rate $R_{\mathrm{AB}}$ with the dynamic phase $k_{0}\left(x_{s}+x_{i}\right)$ is of the form shown by Franson [20] to lead to a violation of a CHSH-Bell inequality. Bell 


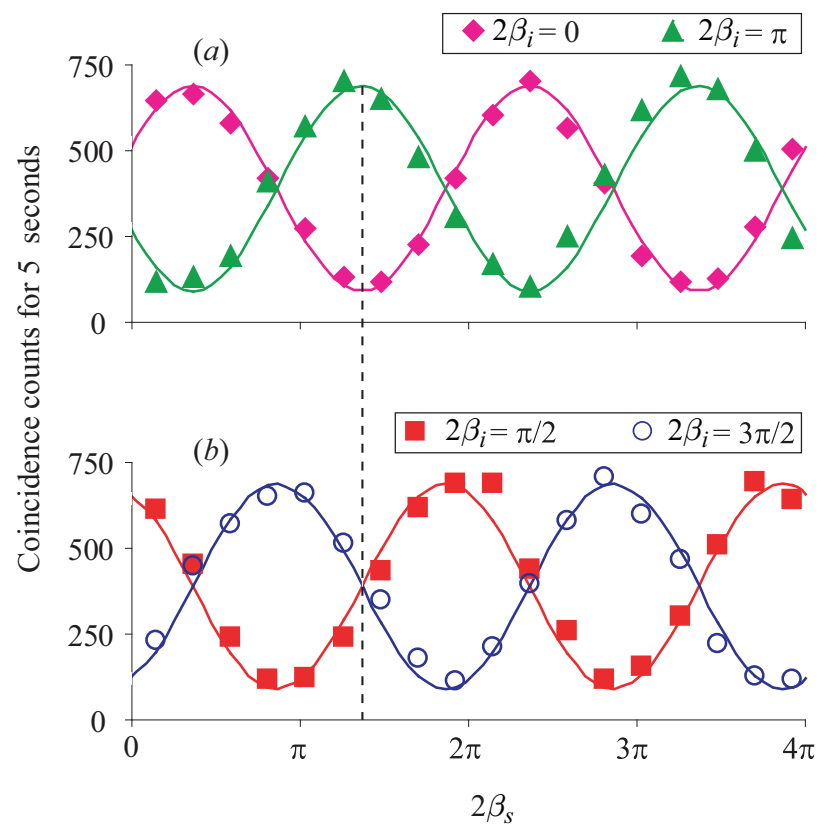

FIG. 3: (color online). Measured number of coincidence counts as a function of $2 \beta_{s}$, the geometric phase of signal photon in alternative 1 , for four different fixed values of $2 \beta_{i}$, the geometric phase of idler photon in alternative 1 . (a) $2 \beta_{i}=0$ and $\pi ;(b) 2 \beta_{i}=\pi / 2$ and $3 \pi / 2$. The solid lines are sinusoidal fits. The distance of the dashed line from the origin is a measure of the fixed value of $k_{0}\left(x_{s}+x_{i}\right)$ to within the period $2 \pi$.

inequality violations based on dynamic phase have been reported in many experiments $[21,22,26]$. Similarly, we note that when the dynamic phase $k_{0}\left(x_{s}+x_{i}\right)$ is held fixed and the geometric phase $2 \beta_{s}+2 \beta_{i}$ is varied, the nature of the variation of the coincidence rate $R_{\mathrm{AB}}$ is still of the form to lead to a violation of the CHSH-Bell inequality, but this time based solely on geometric phase. We next describe our experimental procedure for establishing a violation of this inequality.

The experimental setup was initially aligned such that the distances of the three mirrors from the crystal were all equal to within a millimeter, and thus the condition $|\Delta L|=\left|x_{s}+x_{i}\right| \ll l_{\text {coh }}^{p}$ was satisfied. The idler mirror position was then scanned to observe fringes in the coincidence count rate as a function of $x_{i}$, and it was fixed at a position around which the observed fringe visibility was maximum. At this position, $x_{s}$ and $x_{i}$ were equal to within a few microns and thus the condition $\left|\Delta L^{\prime}\right|=\left|2 x_{i}-2 x_{s}\right| \ll l_{\text {coh }}$ was adequately satisfied. Next, the quarter-wave plate $\mathrm{Q}_{\mathrm{i} 2}$ was successively fixed at four different values of $2 \beta_{i}: 0, \pi / 2, \pi, 3 \pi / 2$. For each value of $2 \beta_{i}$, coincidence counts were measured as a function of $2 \beta_{s}$. Fig. 3 shows the number of coincidence counts plotted against $2 \beta_{s}$ for four different values of $2 \beta_{i}$.

The fringe visibilities shown in these plots are approximately $77 \%$. An experimental demonstration of a viola- tion of a CHSH-Bell inequality requires that the visibility of coincidence fringes be greater than $70.7 \%[16,29]$. The value of the Bell parameter $|\mathrm{S}|$ is determined by the visibility of the coincidence fringes [19, 22]. The sinusoidal coincidence fringes observed in our experiment as functions of $2 \beta_{s}$ for four given values of $2 \beta_{i}$ with visibilities of approximately $77 \%$ imply that the magnitude of the Bell parameter $|\mathrm{S}|$ is approximately $2.18 \pm 0.04$. Therefore, these measurements show a violation of a Bell inequality by approximately 5 standard deviations. A Bell inequality acts as an entanglement witness, and its violation verifies entanglement $[26,30]$. Thus, these results demonstrate that energy-time entanglement can be explored using geometric phases of the signal and idler photons. In our experiment, coincidences were collected for only 5 seconds. Our choice of the coincidence collection time was limited by the overall stability of the interferometer. By collecting coincidences for a longer period of time, a violation with an increased number of standard deviations can be achieved.

Although $77 \%$ visibility is sufficient to show a Bell inequality violation, certain quantum information protocols require visibilities closer to $100 \%$. The main reason for low visibility in our experimental setup is the imperfect overlap of the two interfering two-photon modes. This is caused by the relatively large divergences of the signal and idler modes in alternative 1 compared to their divergences in alternative 2 . This effect could be minimized by using a single spherical mirror, with its center of curvature located at the crystal, for reflecting the pump, signal and idler modes [36]. Another reason for low visibility is the unequal coincidence count rates in the two alternatives. This problem can be taken care of by inserting a variable neutral density filter into the pump beam path between the crystal and the pump mirror. The above factors have also been noted to cause low visibilities in energy-time entanglement experiments based on dynamic phase [22]. We believe that by using customized experimental setups, visibilities closer to the theoretical maximum of $100 \%$ should be achievable.

Geometric phase has found many applications in optics [31, 32]. One of the distinguishing features of geometric phase is its non-dispersive nature. Dynamic phase is introduced by changing the optical path length, and it remains inversely proportional to the wavelength. However, geometric phase is a topological phase and does not depend directly on the wavelength. The nondispersive nature of geometric phase has been demonstrated in white-light interference using achromatic wave plates [33].

For quantum information science, one potential benefit of using geometric phase could be in exploring the energy-time entanglement of ultrabroadband PDC sources $[34,35]$. For such sources, the signal-idler coherence length $l_{\text {coh }}$ remains so small that the visibility of two-photon fringes - which is the magnitude of the prod- 
uct $\gamma(\Delta L) \gamma\left(\Delta L^{\prime}\right)$ - does not remain constant over the variations of $\Delta L^{\prime}$ that are of the order of a wavelength. Therefore, with such sources, dynamic phase is unsuitable for performing Bell inequality violation experiments. Geometric phase, on the other hand, is non-dispersive. Changing geometric phase does not change the optical path lengths. Therefore, the two-photon fringe visibility remains constant as a function of the phase introduced geometrically, and this makes geometric phase particularly suitable for exploring the energy-time entanglement of ultrabroadband PDC sources.

Another benefit of using geometric phase lies in the ease of introducing very small phase shifts. In contrast with dynamic phase, where motorized translation stages are employed, geometric phase is quite easily manipulated by using rotating wave plates. These features of geometric phase may also aid in the construction of hyperentangled states [24-26] with energy-time as one of the degrees of freedom.

In conclusion, we have observed a violation of the Bell inequality for energy and time based purely on the geometric-phases of the signal and idler photons produced by parametric down-conversion. We have thus shown that energy-time entanglement can as well be explored using the geometric phases of the signal and idler photons. These results provide an additional means by which entanglement can be manipulated, and therefore they may have important practical implications for quantum information science.

We gratefully acknowledge financial support through a MURI grant from the U.S. Army Research Office and through an STTR grant from the U.S. Air Force Office. We thank John Sipe, Petros Zerom and Heedeuk Shin for useful discussions.

[1] M. V. Berry, Proc. Roy. Soc. (London) A 392, 45 (1984).

[2] S. Pancharatnam, Proc. Indian Acad. Sci. A44, 247 (1956), reprinted in Collected Works of S. Pancharatnam, edited by G. W. Series (Oxford Univ. Press, New York, 1975).

[3] S. Ramaseshan and R. Nityananda, Curr. Sci. (India) 55, 1225 (1986).

[4] M. V. Berry, J. Mod. Opt. 34, 1401 (1987).

[5] R. Bhandari and J. Samuel, Phys. Rev. Lett. 60, 1211
(1988).

[6] T. H. Chyba, L. J. Wang, L. Mandel and R. Simon, Opt. Lett. 13, 562 (1988).

[7] A. Tomita, and R. Y. Chiao, Phys. Rev. Lett. 57, 937 (1986).

[8] P. G. Kwiat and R. Y. Chiao, Phys. Rev. Lett. 66, 588 (1991).

[9] J. Brendel, W. Dultz, and W. Martienssen, Phys. Rev. A 52, 2551 (1995).

[10] D. V. Strekalov and Y. H. Shih, Phys. Rev. A 56, 3129 (1997).

[11] T. P. Grayson, J. R. Torgerson, and G. A. Barbosa, Phys. Rev. A 49, 626 (1994).

[12] B. Hessmo and E. Sjöqvist, Phys. Rev. A 62, 062301 (2000)

[13] E. J. Galvez, M. Malik, and B. C. Melius, Phys. Rev. A 75, 020302(R) (2007).

[14] J. S. Bell, Physics (Long Island City, N.Y.) 1, 195 (1964).

[15] D. Bohm, Phys. Rev. 85, 166 and 180 (1952).

[16] J. Clauser, M.A. Horne, A. Shimony, and R. A. Holt, Phys. Rev. Lett. 23, 880 (1969).

[17] Z. Y. Ou and L. Mandel, Phys. Rev. Lett. 61, 50 (1988).

[18] Y. H. Shih and C. O. Alley, Phys. Rev. Lett. 61, 2921 (1988).

[19] J. G. Rarity and P. R. Tapster, Phys. Rev. Lett. 64, 2495 (1990)

[20] J. D. Franson, Phys. Rev. Lett. 62, 2205 (1989).

[21] J. Brendel et al., Phys. Rev. Lett. 66, 1142 (1991).

[22] P. G. Kwiat, A. M. Steinberg, and R. Y. Chiao, Phys. Rev. A 47, R2472 (1993).

[23] D. V. Strekalov et al., Phys. Rev. A 54, R1 (1996).

[24] C. Cinelli et al., Phys. Rev. Lett. 95, 240405 (2005).

[25] T. Yang et al., Phys. Rev. Lett. 95, 240406 (2005).

[26] J. T. Barreiro, N. K. Langford, N. A. Peters, and P. G. Kwiat, Phys. Rev. Lett. 95, 260501 (2005).

[27] A. K. Jha, M. N. O'Sullivan, K. W. C. Chan, R. W. Boyd, Phys. Rev. A 77, 021801(R) (2008).

[28] T. J. Herzog, J. G. Rarity, H. Weinfurter, and A. Zeilinger, Phys. Rev. Lett. 72, 629 (1994).

[29] P. G. Kwiat et al., Phys. Rev. Lett. 75, 4337 (1995)

[30] B. M. Terhal, Phys. Lett. A 271, 319 (2000).

[31] P. Hariharan, in Progress in Optics, edited by E. Wolf (North-Holland, Amsterdam, 2005), Vol. 48, p. 149.

[32] R. Bhandari, Phys. Rep. 281, 1 (1997).

[33] P. Hariharan, K. G. Larkin, and M. Roy, J. Mod. Phys. 41, 663667 (1994).

[34] S. Carrasco et al., Opt. Lett. 31, 253 (2006).

[35] M. B. Nasr et al., Phys. Rev. Lett. 100, 183601 (2008).

[36] C. Cinelli et al., Phys. Rev. A 70, 022321 (2004); G. Vallone, Phys. Rev. Lett. 98, 180502 (2007). 